\title{
Protecting Source Location Privacy in IoT Enabled Wireless Sensor Networks: the Case of Multiple Assets
}

\author{
Manjula R, Senior Member, IEEE, Tejodbhav Koduru, and Raja Datta, Senior Member, IEEE
}

\begin{abstract}
A major limitation to the use of Wireless Sensor Networks (WSNs) in asset monitoring applications is the security and privacy concerns, particularly the privacy of source location information. In this paper, we develop two phantom routingbased solutions to provide source location privacy for the case of multi-source/asset scenario- the case that has received very little attention in the literature. The idea of phantom routing is to relay the packets to a distant node in a random fashion to obfuscate the traffic flows and confuse the attacker. The first technique PRBRW uses a combination of backward random walk and a greedy forwarding approach to route the packets to the base station (BS). Although the first approach has better performance improvements in terms of capture ratio and safetyperiod it hampers the lifetime of the network and has a poor entropy metric. To better this problem, an improved phantom routing scheme PRLPRW is proposed. The second technique has three phases: pure random walk, $L$-walk, and greedy walk. This technique performs well in terms of capture-ratio, safety period, and entropy metrics. The improvement in network lifetime is 10 -folds and entropy is 477-folds when compared with PRBRW. The performance is evaluated using the developed analytical models and compared with the base-line protection-less scheme SRP. It is observed that PRBRW and PRLPRW respectively have 60- and 73-fold improvement in terms of capture ratio when compared with SPR. Whereas existing PRPRW and FRW techniques respectively have only 54- and 34-fold improvements.
\end{abstract}

Index Terms-Source Location Privacy, WSN, multi Sources, Passive Attacker, Evaluation.

\section{INTRODUCTION}

$\mathbf{M}$ ilitary strategies are trying to hone their battlefield tactics by adopting Internet of Things (IoT) enabled Wireless Sensor Network (WSNs) [1]-[3]. The deployment of sensors, unmanned aerial vehicles, tiny robots are envisioned to transform the military personnel on the battlefield [4]. Since this is the era of the digital age, the US Army Research Laboratory (ARL) is foreseeing the usage of these smart devices by their soldiers so that they all are connected to the militarys digital communication networks [5] and also know their health status [3], [6]. The aim is to develop a connected network to monitor the soldiers. Specifically, the job of the sensor nodes is to report the health status and whereabouts of the soldiers to a central controller known as the base station (BS). The base station is situated in areas that could be connected to the internet (via IoT system), whereas the sensor nodes are deployed in regions where internet facility is not available [7]. However, this comes with unwelcoming consequences. The connectedness and use of technology bring in a list of

Manjula R is with the Department of Computer Science and Engineering, SRM University AP, Amaravati, Guntur - 522502, INDIA. e-mail: rajamanjula12@gmail.com

Tejodbhav Koduru is with the Department of Computer Science and Engineering, SRM University AP, Amaravati, Guntur - 522502, INDIA. email: tejkodur@gmail.com

Raja Datta is with the departments of GSSST, Indian Institute of Technology Kharagpur, West Bengal- 721302, INDIA.e-mail: rajadatta63@gmail.com risks [7], [8]. For instance, equipping the soldiers' uniforms with Wi-Fi enabled devices could give the enemies many new digital targets. A malicious hacker can compromise the sensors to fake chemical attacks (active attacks) and fool the military men or direct a tank away from their combatants. The attacker can exploit the wireless nature of the communication links by eavesdropping on the signals (passive attacks) and backtrack to the source of information. The attacker finally reaches the soldier or combat vehicle and thus destroys them. These attacks are highly dangerous as losing the assets (military personnel) is a great loss to a nation.

Several solutions exist in the literature that could help counter such attacks in WSNs, a detailed survey is given in [9], [10]. One research area that has gained the attention of the researchers, recently, is the location privacy of the assets (we also alternatively use the terms events or sources to indicate the assets). For example, location of the soldiers in battle field or endangered species in wildlife protection applications.

\section{A. Motivation}

Source Location Privacy (SLP) deals with concealing the source location information, of assets from unauthorized personnel such as attackers but accessible to only authorized personnel [11]. The existing solutions are broadly categorized into: i) Random Walk based solutions [11]-[17] and ii) Fake Source-based solutions [17]-[23]. In random walk based solutions, the information packets are relayed randomly for certain number of hops, starting form the source node, and then delivered to the BS using greedy walk approach (shortest path). Unlike the shortest path routing technique, the random walk based schemes try to obfuscate the traffic pattern so that the traffic analysis by the attacker(s) becomes difficult. In fake source or fake packet based solutions, either the source node(s) or any random node(s) in the network generate fake packets to add ambiguity to the traffic distribution and thus make the traffic indistinguishable to the attackers. Fake-sources/fakepackets based SLP preservation techniques, though provide better performance in terms of privacy, are not suitable for energy-constrained networks. We, therefore, focus on Random Walk (RW) based solutions that are gaining more attention in recent days.

The majority of the work on SLP has been developed to counter the attacker(s) for the case of a single source scenario. However, providing SLP for the case of multi-source scenario-the real-life scenarios has been given less attention. To date and to the best of our knowledge, it is found that the only works that have developed SLP preserving solutions for the multi assets scenarios are seen in [13] and [24]. To fill this gap, we aim to study the performance of SLP preservation solutions (both existing and proposed solutions) developed for WNSs when multi-sources co-exist. 


\section{B. Problem Statement}

The only work that deals with providing fake-source based SLP solutions for the multi-asset scenario is seen in [24]. One cannot guarantee that solutions developed for the case of single-source scenario will also do well for multi-source cases. To know the truth of this observation, the authors in [24] have studied an existing solution [25] and reported that the fake-packet based SLP solution performs well when there is a single source in the network. However, when it comes to the case of protecting source location privacy for multi-asset scenarios the technique performs poorly. They have observed through simulations that no SLP is a better choice when there are multiple sources in the network.

The only work in the literature that has developed a solution to protect multiple assets using the random walk technique is given in [13]. The work assumes that only one source is active while reporting the information to the BS and the rest of the sources are dormant. This assumption effectively boils down the problem to the case of protecting only a single source at any given time instance. Protecting a single source might be a hypothetical scenario. To be realistic and more practical oriented, we intend to study the effect of SLP preservation techniques when multiple sources co-exist in the network, using random walk-based solutions

One may argue that the presence of multiple sources in itself inherently provides routing path obfuscation, then why do we need SLP preservation solutions? The authors in [26] have mathematically proved that the presence of multiple sources alone might not give inherent routing path obfuscation (or privacy). Added to this, the authors in [24] have also shown that fake packet-based SLP solutions do not perform well when multiple sources co-exist in the network.

Motivated by these observations and to answer the longawaited question: "What will be the effectiveness of random walk-based SLP preservation techniques in multi-source settings?", we aim to study this scenario.

\section{Contributions and Organizations:}

To study the impact of random walk-based SLP preservation techniques in multi-source settings, we develop two randomwalk based solutions to mitigate the said issues: i) Phantom Routing-based Backward Random Walk (PRBRW), and ii) Phantom Routing based L-Path Random Walk (PRLPRW). The PRBRW technique comprises two phases. The first phase, backward random walk, is intended to divert the information packet away from the source and the second phase is to deliver the packet to the BS in a greedy fashion. Although we have seen improvements in the performance metrics such as capture ratio and safety-period (defined in Sec. VI), the entropy and the network lifetime metrics are reduced. To mitigate this issue, we proposed the PRLPRW technique. This solution comprises three phases. The first two phases, namely the pure random walk phase and the L-path phase, are intended to take the packet away from the source node and the third phase is meant to deliver the packet to the BS in a greedy approach. In the PRBRW technique, the packet in the first phase is always sent in the backward direction away from the source node. Since the backward random walk is confined to a narrower region, the information packets tend to follow almost the same set of paths. This drains the energy at a faster rate in all those nodes that present in this region and hence reduces the network lifetime and entropy. The second method overcomes this issue by spreading the network traffic widely across the network and thus improves the entropy and lifetime of the network. We evaluate the effectiveness of the proposed solutions using the developed analytical models. In additions, the performance improvements of the proposed techniques are measured by comparing them with the representative techniques namely, Phantom Routing based Pure Random Walk (PRPRW) [17] and Forward Random Walk (FRW) [14], through simulations.

In summary, the contributions of the work are:

- Develop two new phantom routing schemes to protect source location privacy of multiple assets in IoT enabled WSNs.

- Provide detailed analysis, through simulations and math models, of the performance of the existing Random Walk based solutions and the proposed techniques.

- Provide insights, through observations, into the effect of source location privacy for the case of mobile assets in the network-suggestion for future researchers.

The rest of the paper is organized as follows: In section II, we give the overview of the existing SLP techniques, on selected solutions only. In Sec. III and Sec. IV, we explain the network model and attacker model, respectively, considered in our work. We then present the proposed solutions, the metrics considered for the work and their performance analyses in Sec. V, Sec. VI, and Sec. VII respectively. Finally, we conclude the work in Sec. VIII.

\section{LITERATURE REVIEW}

Here, we present the existing literature on SLP preserving protocols developed for the case of multiple assets scenario. We also brief about the solutions PRPRW and FRW (designed for protecting a single asset) that we use in this work for comparison purposes.

The author's work in [13] is the pioneering work on SLP preserving solution in WSNs using the random walk-based approach for the case of multiple asset scenario. In this work, the author assumes that only one source is active at a time while other sources are dormant. The sensor nodes that have detected the asset (say an endangered animal) send an alert message to two-hop neighbors. All the nodes within this two-hop distance declare themselves as Alert-Zone nodes. These zones are created so that the packets sent by other source nodes do not enter these zones, which would otherwise attract the back-tracing attacker to any one of the assets. After detecting the asset, the source node prepares information packets about the presence of the asset and sends it to the BS. The routing protocol is initially in a greedy approach. At every intermediate node, a random number is generated between 0 and 1 . If the value of the random number is less than the prescribed threshold value, then the routing enters into a biased random walk model. Else it continues to follow a greedy approach. In the meantime, if a node encounters a neighbor that belongs to the Alert-Zone then it avoids that neighbor from being chosen as the forwarding neighbor. This way, the information packets are delivered to the BS. However, the solution does not give details on how to put other source nodes into dormant mode while only one source is made active. This gives scope to explore this approach further.

The author's in [24] have tested the effect of fake packetbased SLP protection for the case of multiple source scenario. To study the impact of fake packets or fake sources, the authors adopt the SLP routing protocol developed in [25]. The work demonstrates the effect of these algorithms for the cases of a single source, two sources, three sources, and four sources in 
the network (co-exist at the same time). The study concluded that protection-less communication (no privacy) is better than SLP based communication when fake packets are employed, to obfuscate the attacker. The paper concludes by stating an open challenge to the prospective researchers to develop efficient solutions for the case of multiple assets scenario that employ fake packets or fake sources.

Phantom Routing based Pure Random Walk (PRPRW) solution was suggested, for the first time, in [11]. This technique is designed to deal with a single source scenario. The idea is to initially send the information packet from the source node to a phantom node in a random fashion. Once the packet reaches the phantom node, it sends the packet to the BS using the min-hop routing strategy. Initially, the source sets the packet with a TTL value that is updated (decremented) by every intermediate relaying node. The packet relaying is on the random selection of a neighbor node. The random sending of the packet continues as long as the TTL value is non-zero. Once this value is zero, it is sent to the BS using min-hop routing. The node at which the TTL value is zero is termed as the phantom source.

In the Forward Random Walk (FRW) approach [14], every node in the network divides its neighbors into three groups. One group has nodes that are closer to the BS than itself. The second group has those neighbors whose depth to the BS is the same as that of itself and the rest are grouped into the third category. The nodes from the first and the second group together form the forwarding list. Whenever a node has a packet to be relayed, it randomly chooses one of the neighbors to form this forwarding list and relays the packet to that node. This process repeats until the packet reaches the BS. This technique also deals with a single source scenario.

\section{NETWORK MODEL}

The sensors are deployed in the target field as shown in Fig. 1. Each sensor node (circles) comprises a transceiver to communicate the sensed information; sensors to monitor the surrounding area and to detect or locate the target [27]; microprocessor/microcontroller; memory \& power unit. We also consider that the asset(s) of interest are tagged with Radio Frequency Identification (RFID) transponder while the sensor nodes are equipped with the RFID reader. Whenever the asset comes within the sensing range of a sensor node, the RFID tag on the asset communicates with the reader on the sensor node. The sensor node then sends this information to the BS using the developed SLP privacy preservation techniques. In our scenario, we assume that the sensor node's sensing range $r_{s}$ and its communication range $r_{c}$ are both equal. All the communications in the network are assumed to be secured and reliable. The network considered is a square deployment model with nodes arranged regularly with the distance between any two adjacent nodes being $r_{s} \sqrt{2} / 2$, as shown in Fig. 1 . This arrangement gives each node eight neighbors that are further grouped into three categories: i) Closer Set neighbors, ii) Equal Set neighbors, and iii) Farther Set neighbors (see Sec. V for details). There is only a single base station (BS) located at the center of the deployment model as shown in Fig. 1, whose position information is known to everyone, including the attacker. However, there are multiple sources in the network whose location information must be known only to authorized personnel and not the attacker.

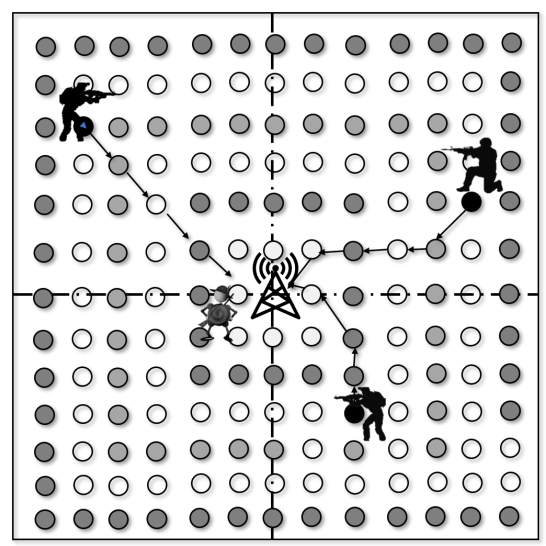

Fig. 1: Network and Attack Model

\section{ATtACKER MODEL}

We consider a single and mobile attacker that attacks the network at the BS, as it is the focal point for entire network traffic. The attacker is equipped with resources such as a spectrum analyzer, laptop, etc. The sensing range is the same as that of the sensor nodes. Due to the limited communication range, the attacker has only a local view of the network traffic. The attacker performs passive attacks such as eavesdropping attacks and then analyzes the traffic details to gain contextual information. Initially, the attacker starts at the BS and waits there until it senses a signal (a packet) coming towards the $\mathrm{BS}$, as shown in Fig. 1. After receiving the packet it moves one hop to the next node, in the direction of the signal arrival and away from the BS, and stays there until it receives a new packet from a new direction (never retreats). This way the attacker keeps backtracking the messages hop-by-hop until it reaches any one of the source nodes. The game is over.

\section{Proposed Methods}

The proposed solutions comprise of two phases: i) Initialization Phase and ii) Operational Phase.

\section{A. Initialization Phase}

In this phase, the BS initiates a flooding protocol for configuration purpose, after the sensor nodes and BS are deployed in the network. The flooding message contains a depth field that is initially set to zero by the BS. A node upon receiving this flooding message updates its routing table with the depth value that is specified in the packet and the sender's details. It then adds one to the depth field in the packet and includes its details such as identity (ID), depth value, initial energy, position coordinates, etc, in the received packet. The updated packet is then rebroadcasted into the network. This process repeats until the flooding has reached the network boundary. The nodes that are within the BS's radio range will have the depth of value one, the nodes surrounding these nodes will have the depth value of two, and so on. At this stage, every node in the network knows its position in the network (w.r.t BS position), has knowledge on its neighbors' information such as the number of neighbors, their IDs, neighbors' distance to the BS (in terms of depth value), initial battery energy, and other security-related information. The security-related information is necessary for securing the message content. The details regarding safeguarding message content are beyond the scope of the work. 


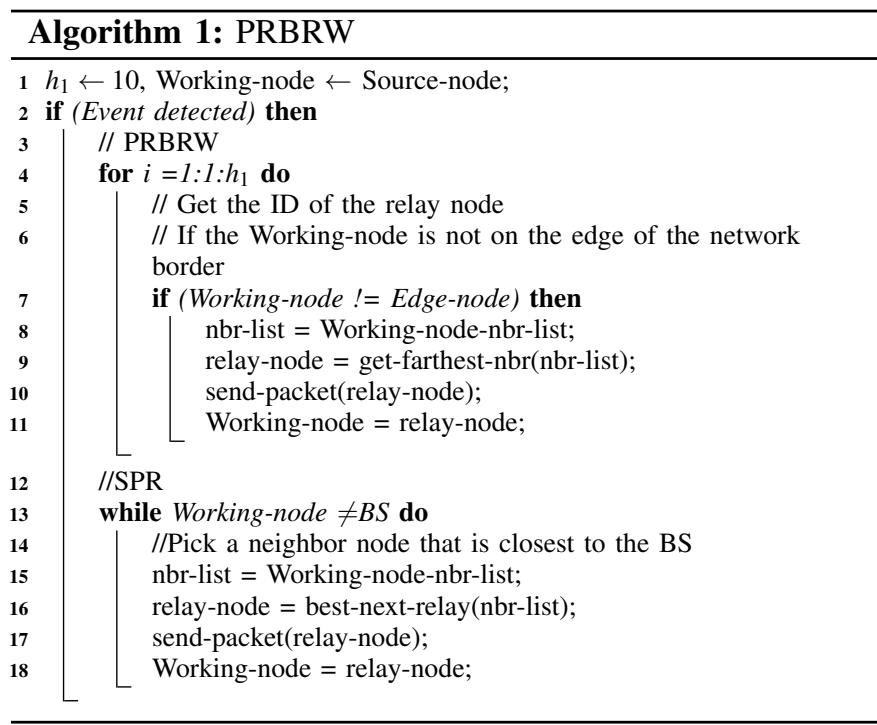

\section{B. Operational Phase}

During this phase, the proposed algorithms work.

1) Phantom Routing-based Backward Random Walk:

In this technique, every node divides its neighbors into three groups as forwarding neighbors, equal neighbors, and closer neighbors based on the depth value of the neighbors. A sensor node groups all those neighbors whose depth value is smaller than its depth value and puts them in a closer-set. Similarly, it groups all those neighbors whose depth value is equal to its depth value into a equal-set. The rest of the neighbors are grouped into farther-set. The nodes in equal-set and fartherset are combined together to form the backward-set. This technique works in two phases that are explained as follows:

- Backward Random Walk phase (PRBRW): Upon detecting an event, the source node randomly chooses a neighbor from the backward-set and relays the information packet to that node. This packet has a Time To Live (TTL) field which is set to $h_{1}$ hops. A neighbor node that receives this packet first checks the TTL value. If this value is not zero, then it decrements the value of $h_{1}$ by one, randomly selects a neighbor from its backward-set, and relays the packet to that neighbor. This process repeats as long as $h_{1}$ is not zero. Once the value of $h_{1}$ equals zero, the message is sent using phase two. The node that holds the packet when $h_{1}$ is zero is termed as phantom node or phantom source.

- Shortest Path Routing Phase: The phantom node sends the packet to the BS using the greedy approach. In this phase, every intermediate node forwards the message only to a neighbor node which has the least depth value to the BS. This effectively forms the shortest path between the phantom node and the BS. This phase is intended to converge the random walk at the BS and also it helps save energy.

2) Phantom Routing-based L-Path Random Walk (PRL$P R W)$ :

PRBRW though performs well in privacy aspect it has poor performance in terms of entropy and network lifetime metrics. Our second technique PRLPRW aims at improving these two limitations by widely spreading the network traffic paths in the network. PRLPRW technique consists of three phases:

- Pure Random Walk Phase: Upon detecting an event, the source node enters into pure-random walk phase where it randomly chooses one of its neighbor with equal

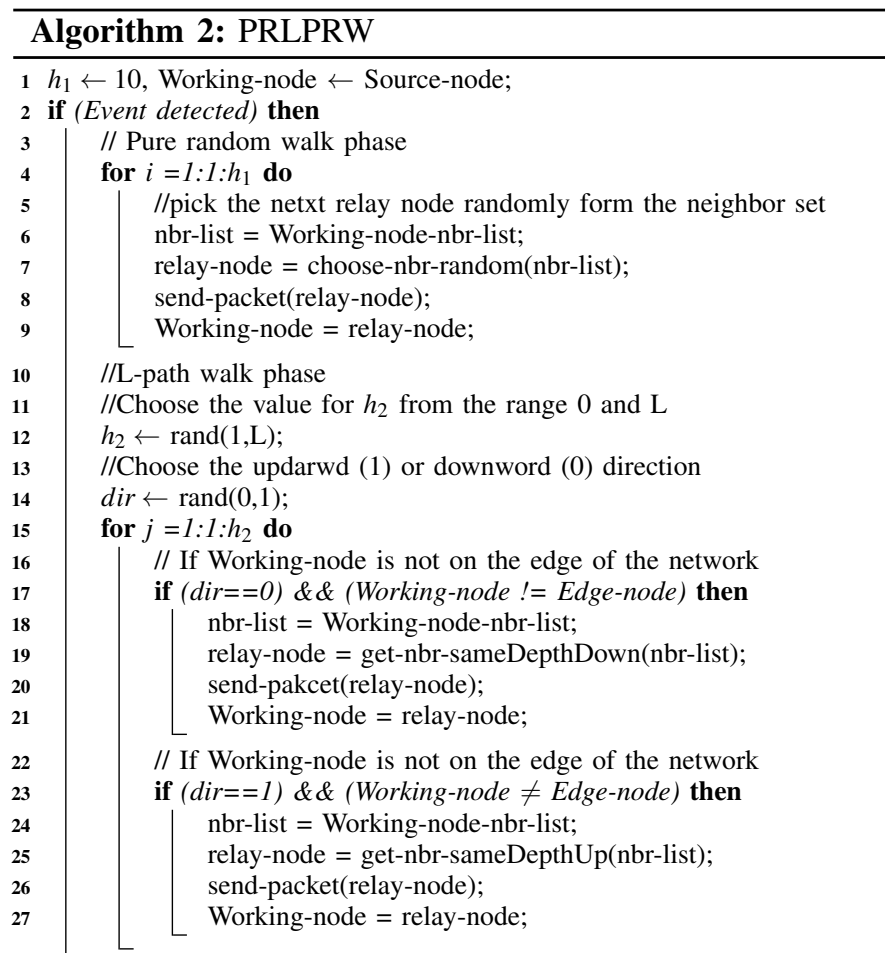

//Choose the new value for $h_{2}$ from the range 0 and $\mathrm{L}$ $h_{2} \leftarrow \operatorname{rand}(1, \mathrm{~L})$

//Choose the left (1) or right (0) direction

$\operatorname{dir} \leftarrow \operatorname{rand}(0,1)$;

for $j=1: 1: h_{2}$ do

// If Working-node is not on the edge of the network

if $($ dir $==0) \& \&$ (Working-node $!=$ Edge-node) then nbr-list $=$ Working-node-nbr-list ;

relay-node $=$ get-nbr-sameDepthRight(nbr-list); send-pakcet(relay-node);

Working-node = relay-node;

// If Working-node is not on the edge of the network if $($ dir $==1) \& \&$ (Working-node $\neq$ Edge-node $)$ then nbr-list $=$ Working-node-nbr-list; relay-node $=$ get-nbr-sameDepthLeft(nbr-list); send-pakcet(relay-node); Working-node = relay-node;

//SPR phase

while Working-node $!=B S$ do

//Pick a neighbor node that is closest to the BS

nbr-list $=$ Working-node-nbr-list;

relay-node $=$ best-next-relay(nbr-list);

send-packet(relay-node);

Working-node $=$ relay-node;

probability and relays the packet to that neighbor. The source also sets the time to live (TTL) field in the packet to a value $h_{1}$. The neighbor node receives the packet; decrements the TTL value by one, and randomly selects a neighbor with equal probability to relay the packet. This process repeats for $h_{1}$ times. After completing $h_{1}$ random hops, the packet enters into the second phase. The node at which the TTL value becomes zero is termed as phantom node. The phantom node now invokes the L-walk phase.

- L-Walk Phase: In this phase, the packet moves in vertical and horizontal directions. Combinations of both these vertical-walk and horizontal-walk drive the information packet far away from the main source and the phantom source. At the beginning of this phase, the node takes two decisions: i) Whether to send the message vertically up or down in case of vertical movement, and horizontally 
left or right in case of horizontal movement, and ii) How many hops $h_{2}$ the packet should travel in the chosen direction. The phantom source takes these decisions arbitrarily. The hop count value is a random number chosen from the range 1 and $L$, where $L$ is the side length of the deployment area in terms of the number of hops (i.e., $h_{2}=L / r_{s}$ hops). This value is then updated in the packet before relaying it further. Once the direction (vertical or horizontal) and hop count are chosen by the phantom node, it sends this packet using the L-Walk phase. A neighbor that receives this packet will check for the hop count value and the direction (say vertical direction) specified in the packet. If the hop count value $h_{2}$ is not yet zero, it decrements the hop count value by one and then relays the packet to the next node that satisfies the direction mentioned in the packet. Once the TTL value $h_{2}$ is zero, the node that posses this packet will chose another direction (this time horizontal direction), and reinitializes the TTL value $h_{2}$ with a number randomly chosen from range 1 and $L$ and relays the packet to a neighbor that is located in the desired direction. The process of relaying the packet in the new direction continues as long as the TTL value is not zero. Once the TTL value is zero, the packet enters into phase three.

- Shortest Walk Phase: This phase is the same as the one described in the PRBRW technique.

The algorithms for the proposed techniques are shown in Algo. 1 and Algo. 2 respectively.

\section{Vi. Performance Characterization}

We use the following metrics to measure the performance of SLP techniques:

- Capture Ratio: The capture ratio (also known as capture percentage) is defined as the ratio of the number of trials in which the asset is captured by the attacker before the simulation ends to the total number of trials carried out.

- Average Hops: This metrics helps us measure the average number of hops the data packets take to reach the base station. We develop theoretical models (See Appendix A) to determine the average number of hops data packets take to reach the BS, when sent from source node(s). This metric could also be used to estimate average delay in terms of hops.

- The average number of hops $\overline{H_{1}}$ between the source node and the base station for PRPRW technique is given by:

$$
\bar{H}_{1}=h_{1}+\frac{1}{h_{1}} \sum_{i=1}^{h_{1}} \frac{\sqrt{d_{1}^{2}+\left(i \times r_{s}\right)^{2}}}{r_{s}}
$$

- The average number of hops in forward random walk routing technique (FRW) is estimated using the following equation:

$$
\bar{H}_{2}=n^{\prime} / p_{r}
$$

- The average number of hops $\overline{H_{3}}$ and $\overline{H_{4}}$ in PRBRW and PRLPRW technqiques respectively are caluclated as follows:

$$
\begin{gathered}
\overline{H_{3}}=h_{1}+\frac{\overline{H_{1}}}{2} \\
\overline{H_{4}}=h_{1}+\frac{L}{r_{s} \sqrt{2}}(\sqrt{2}+1)
\end{gathered}
$$

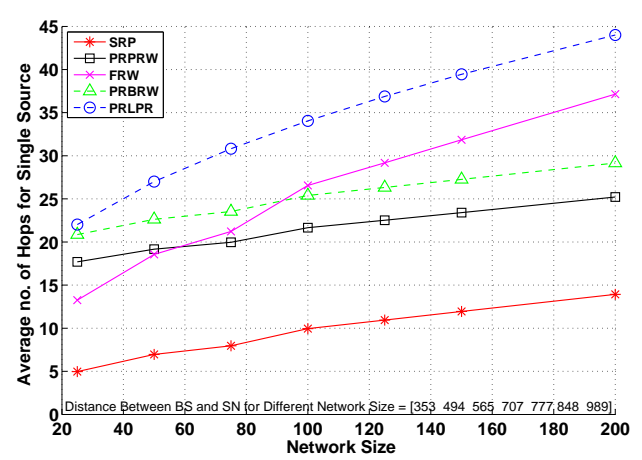

Fig. 2: Average no. of Hops for Single Source Case (Analytically)

Fig. 2 depicts the average number of hops (i.e., the average delay), obtained through analytical simulations, for all the techniques.

- Safety Period: Defined as "the number of packets delivered to the base station before the adversary reaches the source of origin" [11].

- Energy consumption: Energy is expended while processing information packets as well as while transmitting and receiving the packets. We consider the energy spent only during message exchanges among nodes. To compute the energy expenditure we use the models as suggested in [28] and are given as follows:

$$
\begin{aligned}
E_{T x}(l, d) & =E_{\text {elec }} \times l+\varepsilon_{f s} \times l \times d^{2}, \quad d \leq d_{0} \\
& =E_{\text {elec }} \times l+\varepsilon_{\text {amp }} \times l \times d^{4}, \quad d>d_{0}
\end{aligned}
$$

Similarly, the energy consumption for receiving a message of length $l$-bits is given by

$$
E_{R x}(l)=l \times E_{\text {elec }}
$$

where $E_{\text {elec }}$ is the energy losses in the transmit circuit that depends on factors such as digital coding, spreading of the signal and filtering. The amplifier's energy, $\varepsilon_{f s} \times l \times d^{2}$ or $\varepsilon_{a m p} \times l \times d^{4}$, depends on the distance to the receiver and the acceptable bit-error rate. The notations used in the analyses of energy expenditure are given in Table I.

- Entropy or Path Randomness: In information theoretic approach, entropy is used to determine the average amount of uncertainty on given set of information. Later this theory was also used by communication sector to determine uncertainties in network traffic. In this work, we use entropy to measure the amount of randomness of the routing paths created by the random walks. Precisely this metric indicates traffic diversity and is measured using the following equation:

$$
H=\sum_{i=1}^{i=N} p_{i} \log _{2}\left(1 / p_{i}\right)
$$

where $p_{i}$ is the ratio of number of packets forwarded/relayed by a sensor node to the total number of packets sent by the source node(s). If majority of the nodes in the network have forwarded equal number of packets then the value of $p_{i}$ tends to be equal. This implies packets are routed widely across the network thus spreading the traffic. The higher the value of $H$, more random is the network traffic and more difficult will be 
TABLE I: Notations

\begin{tabular}{|c|c|c|c|}
\hline Notations & Description & Notations & Description \\
\hline ID & Identity & $B S$ & Base Station \\
\hline$L$ & Side length of a squared deployment area & $r_{s}$ & Radio range of nodes in WSN (71 units) \\
\hline$h_{1}$ & Maximum no. of hops in random walk & $\bar{H}_{1}$ & Average no. of hops in PRPRW \\
\hline $\bar{H}_{2}$ & Average no. of hops in FRW & $\bar{H}_{3}$ & Average no. of hops in BPRW \\
\hline $\bar{H}_{4}$ & Average no. of hops in PRLPRW & $d_{1}$ & Distance between source node and BS \\
\hline$d_{2}$ & Distance between the phantom node and the BS & $d_{3}$ & Mintance between Source node and Phantom node \\
\hline$D\left(n_{i}\right)$ & Depth of a node $n_{i}$ & $n^{\prime}$ & Energy losses in the transmission circuits \\
\hline$d_{0}=87$ & Distance threshold for different channel models & $E_{e l e c}=50 n J /$ bit & Power amplification energy in multi-path fading model \\
\hline$e_{f_{s}}=10 \mathrm{pJ} / \mathrm{bit} / \mathrm{m}^{2}$ & Power amplification energy in free space model & $e_{a m p}=0.0013 p J / b i t / m^{4}$ & Powe \\
\hline$l=6392$ bits & Length of the message & $d$ & Distance between the sending node and receiving node \\
\hline
\end{tabular}

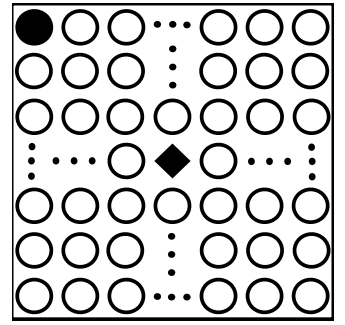

(a) Single Source Node in Second Quadrant

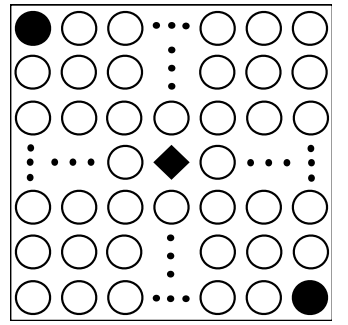

(b) Two Source Nodes in Opposite Quadrants

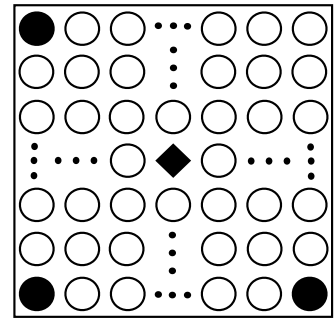

(c) Three Source Nodes in Three Quadrants

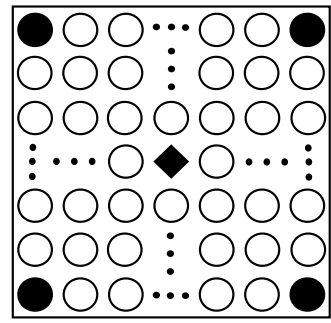

(d) Four Source Nodes in all Four Quadrants

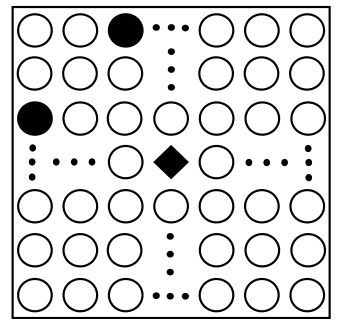

(e) Two Source Nodes in the Same Quadrant

Fig. 3: Network Configurations

the backtracking process of the attacker. $H$ reaches the maximum value only when $p_{i}$ is equal across all the nodes in the network.

- Network Lifetime: It is defined as the period from the beginning of the network operation until the first node runs out of battery energy. It is measured based on the number of packets sent in the network before a node dies.

\section{RESULTS AND DISCUSSION}

In this section, we initially describe the simulation settings and then provide extensive analyses on the results obtained through simulations. Finally, we end this section with a discussion and future research direction. The proposed techniques were compared with the baseline scheme SPR (no privacy protection) and the existing solutions PRPRW [17] and FRW [14] to evaluate the effectiveness in terms of the performance metrics, as given in Sec. V. We implemented all the solutions in the Python programming language.

\section{A. Simulation Settings:}

The network deployment model is as shown in Fig. 1. The radio range is set to 71 units, including the BS's and the adversary's radio range. Each node is loaded with 0.5 joules of energy. Table I shows the notations (along with the descriptions) used in this work.

In the scenarios, we considered 1, 2, 3, and 4 source nodes in the network respectively. These cases are depicted in Fig. 3. The details of each of these source nodes, along with their positions and distance from the BS, as well as the network dimension are given in Table II. In each case, we carried out 100 trials and then took the average of all these 100 trials. Each node sends 60 packets in the entire simulation and the simulation ends when either all the packets are sent or the attacker reaches the source node. Fig. 3a shows the case of a single source in the network. It is located in the left corner and the BS is located in the center of the network. Keeping these positions fixed, the size of the network was varied by increasing the side length of the square deployment area. The values considered are $25,50,75,100,125,150$ and $200 \times 10^{4}$ square meters, respectively. In each of the cases, the number of sensor nodes was also varied as $120,224,288,440,528$, 624,840 respectively. Fig. 3b-3d shows the case of multiple source scenario. For two source nodes case, we consider they are in opposite quadrant of the network as shown in Fig. 3b. Similarly, three and four source nodes cases are as shown in Fig. 3c and Fig. 3d. Fig. 3e shows the case of two sources lying in the same quadrant. The rest of the settings for various trials is similar to the one described for a single source case.

Further, we mention that in all the performance metrics, the graphs shown are averaged over the number of source nodes in the network. This is done to maintain uniformity on the values of the $y$-axis scale. Specifically, the simulations are the average of 100 trials and each result is in turn divided by 2 , 3 , and 4 for two source nodes, three source nodes, and four source nodes cases respectively.

\section{B. Result Analyses}

We study the performance of the proposed SLP techniques and the existing solutions in this section. To understand the behavior of the SLP schemes we have carried out extensive simulations for various network configurations and the analyses of the results thus obtained are given as follows:

\section{1) Capture Ratio}

Fig. 4 shows the plots for the metric capture ratio. The plots are obtained by varying the network size and for each case, we observed the number of trails in which the attacker is successful to know the capture percentage. We observe that the no protection scheme namely, shortest path routing (SPR) has a $100 \%$ capture ratio. Whereas other techniques have a lower capture percentage. The capture percentage decreases as the network size increase. This implies larger is the network size better is the privacy, i.e., safer is the asset. We also 
TABLE II: Details of the Deployment Scenario, Number of Source Nodes, and Their Position in the Network
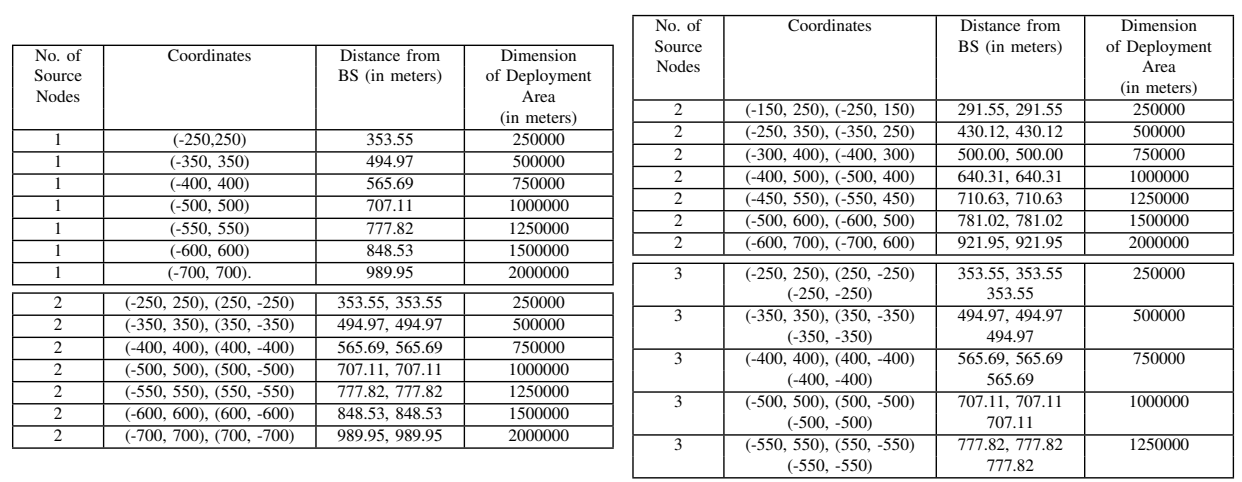

\begin{tabular}{|c|c|c|c|}
\hline $\begin{array}{l}\text { No. of } \\
\text { Source } \\
\text { Nodes }\end{array}$ & Coordinates & $\begin{array}{l}\text { Distance from } \\
\text { BS (in meters) }\end{array}$ & $\begin{array}{c}\text { Dimension } \\
\text { of Deployment } \\
\text { Area } \\
\text { (in meters) }\end{array}$ \\
\hline 3 & $\begin{array}{c}(-600,600),(600,-600) \\
(-600,-600)\end{array}$ & $\begin{array}{c}848.53,848.53 \\
848.53\end{array}$ & 1500000 \\
\hline 3 & $\begin{array}{c}(-700,700),(700,-700) \\
(-700,-700)\end{array}$ & $\begin{array}{c}989.95,989.95 \\
989.95 \\
\end{array}$ & 2000000 \\
\hline 4 & $\begin{array}{c}(-250,250),(250,-250) \\
(-250,-250),(250,250)\end{array}$ & $\begin{array}{l}353.55,353.55 \\
353.55,353.55 \\
\end{array}$ & 250000 \\
\hline 4 & $\begin{array}{c}(-350,350),(350,-350) \\
(-350,-350),(350,350)\end{array}$ & $\begin{array}{l}494.97,494.97 \\
494.97,494.97 \\
\end{array}$ & 500000 \\
\hline 4 & $\begin{array}{c}(-400,400),(400,-400) \\
(-400,-400),(400,400)\end{array}$ & $\begin{array}{c}565.69,565.69 \\
565.69,565.69\end{array}$ & 750000 \\
\hline 4 & $\begin{array}{c}(-500,500),(500,-500) \\
(-500,-500),(500,500)\end{array}$ & $\begin{array}{l}707.11,707.11 \\
707.11,707.11 \\
\end{array}$ & 1000000 \\
\hline 4 & $\begin{array}{l}(-550,550),(550,-550) \\
(-550,-550),(550,550)\end{array}$ & $\begin{array}{l}777.82,777.82 \\
777.82,777.82\end{array}$ & 1250000 \\
\hline 4 & $\begin{array}{l}(-600,600),(600,-600) \\
(-600,-600),(600,600)\end{array}$ & $\begin{array}{l}848.53,848.53 \\
848.53,848.53 \\
\end{array}$ & 1500000 \\
\hline 4 & $\begin{array}{l}(-700,700),(700,-700) \\
(-700,-700),(700,700)\end{array}$ & $\begin{array}{l}989.95,989.95 \\
989.95,989.95 \\
\end{array}$ & 2000000 \\
\hline
\end{tabular}

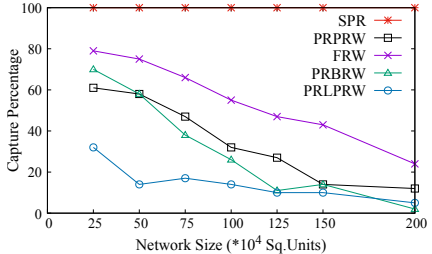

(a) Single Source Node

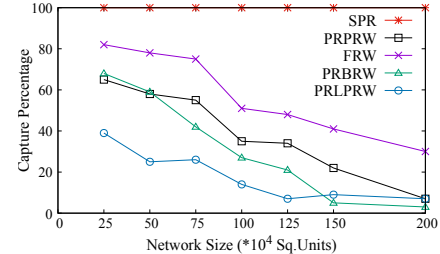

(b) Two Source Nodes

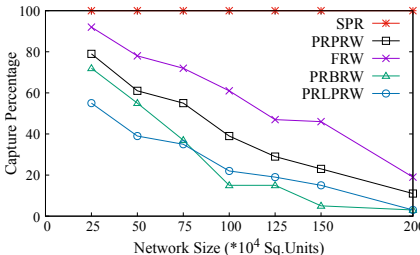

(c) Three Source Nodes

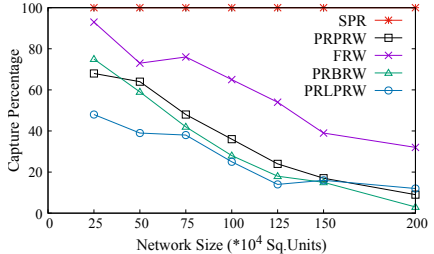

(d) Four Source Nodes

Fig. 4: Capture Ratio

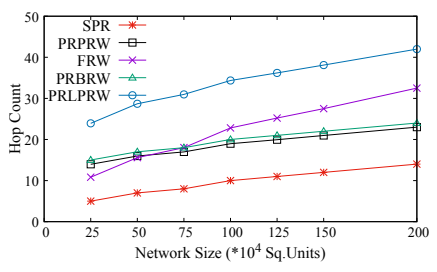

(a) Single Source Node

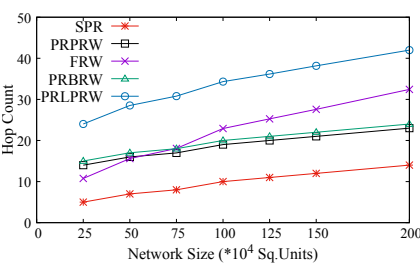

(b) Two Source Nodes

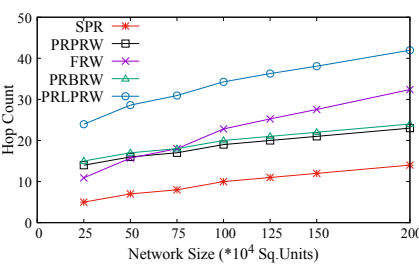

(c) Three Source Nodes

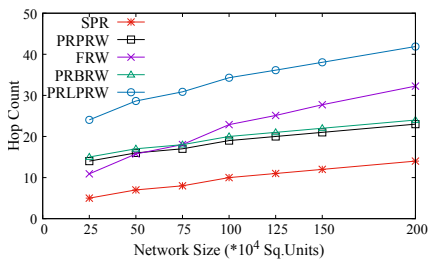

(d) Four Source Nodes

Fig. 5: Average no. of Hops Traversed by the Packets

observe that the capture ratio in the proposed technique is the least when compared with other existing techniques, in all the settings. The low capture ratio in random walk based techniques is because the packets take different paths to reach the BS. As a result of this, the adversary keeps wandering in the network for a longer time period and misses capturing the source within a specified time interval. A noteworthy observation we made in this study is that the network size, location of and the number of source nodes also have an immense effect on the capture percentage of random walk based routing schemes. As the number of source nodes (assets) increases the capture percentage decreases. This implies that the presence of multiple sources itself increases privacy level. However, we stress that the mere presence of multiple sources in the network does not affect capture percentage in the case of the shortest path routing (SPR) technique (protection-less technique). This observation is in-line with the statement that is alluded to in Sec. I-B. This shows that we still need SLP techniques even if there are multiple sources in the network.

\section{2) Hop Count}

The plots for the average number of hops taken by packets to reach the BS for various network sizes are as shown in Fig. 5. The figure shows that as the network size increases, the average number of hops also increases. For small-sized networks, the newly proposed techniques perform well. However, for larger network sizes, PRBRW has a fewer number of hops when compared to the number of hops in the FRW technique. The reason for a fewer number of hops in PRBRW is because as the distance between the BS and source node increases, the divergence of the routing path tends to be narrower when viewed from the BS perspective and the packets tend to follow almost in the same set of paths. Whereas in the case of FRW, the amount of path divergence almost remains the same leading to more hops. However, PRLPRW has more hops compared to all other techniques. The improvement is because the packets are following different routes to reach the BS, these routes are widely spread across the network. Our simulation results are well matching with the analytical results that are presented in Sec. VI, Fig. 2.

\section{3) Safety Period}

Fig. 6 shows the plots for the safety period metric. The safety period is lowest for SPR (the base-line technique) compared to other SLP protocols as it does not add any contribution towards routing path randomness. It is observed that the safety period increases with the increase in network size for all the solutions. Since the distance between the source nodes and the BS increases with the increase in network size, the attacker has to travel a longer distance to reach any one of the sources. The proposed two techniques have better performance compared to the FRW technique because these schemes 


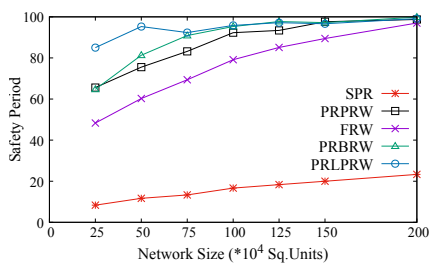

(a) Single Source Node

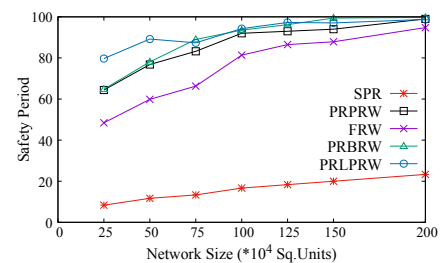

(b) Two Source Nodes

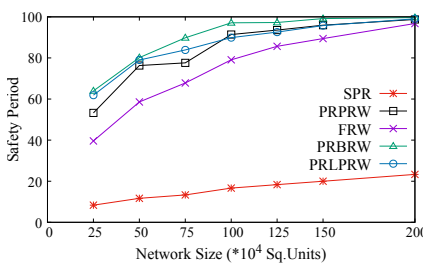

(c) Three Source Nodes

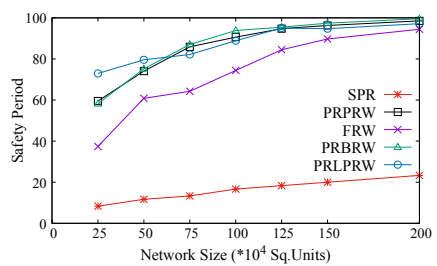

(d) Four Source Nodes

Fig. 6: Safety Period

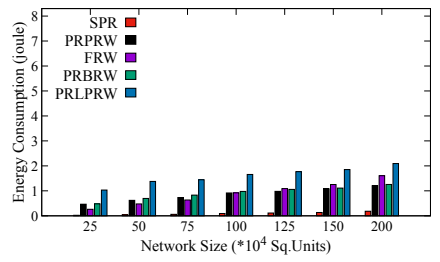

(a) Single Source Node

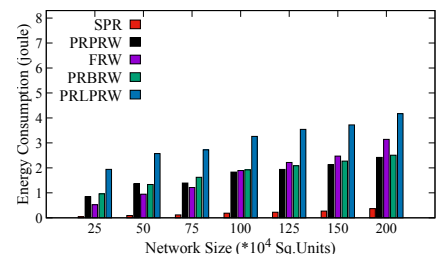

(b) Two Source Nodes

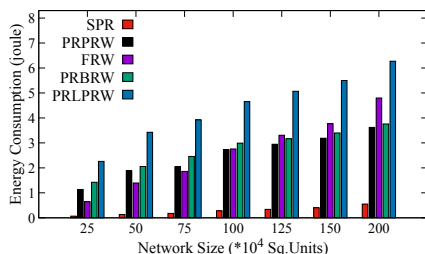

(c) Three Source Nodes

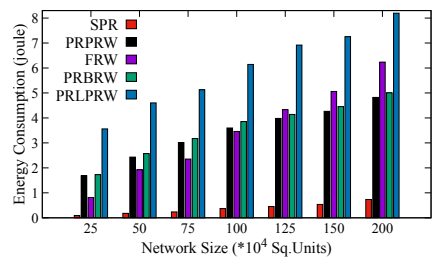

(d) Four Source Nodes

Fig. 7: Energy Consumption

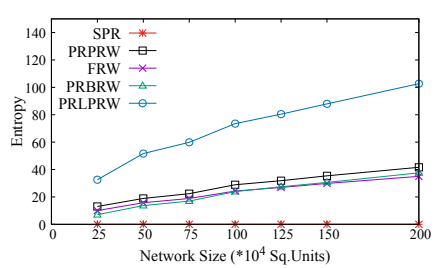

(a) Single Source Node

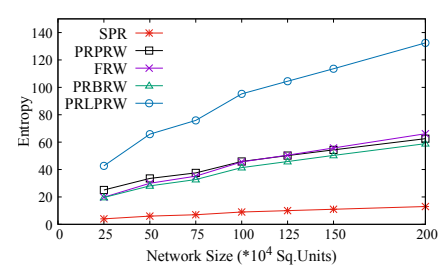

(b) Two Source Nodes

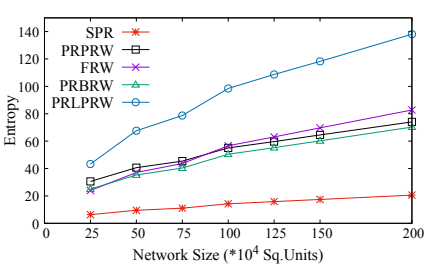

(c) Three Source Nodes

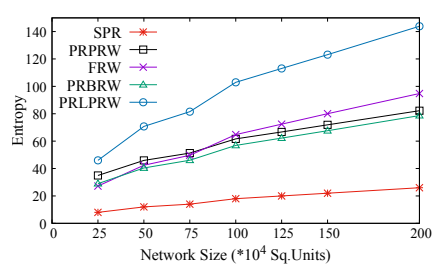

(d) Four Source Nodes

Fig. 8: Entropy

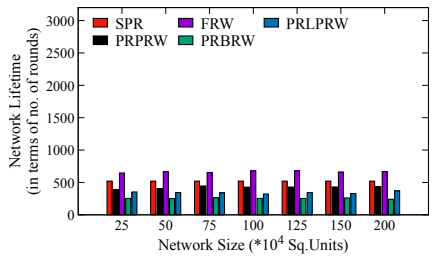

(a) Single Source Node

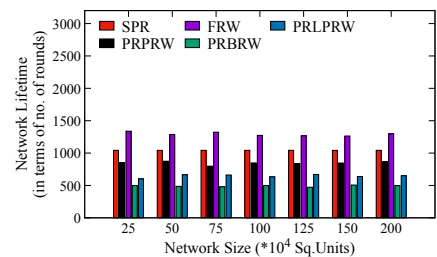

(b) Two Source Nodes

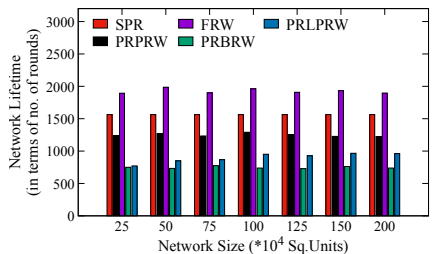

(c) Three Source Nodes

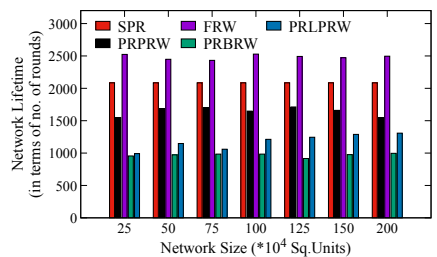

(d) Four Source Nodes

Fig. 9: Network Lifetime

provide better traffic obfuscation. PRPRW performs better than FRW and SPR techniques but does not do well when compared to PRBRW and PRLPRW. Another observation we made is that for small-sized networks, the presence of multiple sources in the network decreases the overall safety period. This again shows that the presence of multiple assets in the network alone will not provide privacy and calls for providing privacy protection schemes.

\section{4) Energy Consumption}

The energy expenditure incurred while delivering the packets to the BS is as shown in Fig. 5. Whenever a node relays a packet or receives a packet it has to expend energy. In our simulation work, we have considered the energy models as given in Eq. 5a and Eq. 5b. It is natural that as the routing paths become longer and more divergent, due to randomness, energy consumption increases while sending packets to the BS. Both the proposed techniques incur extra energy expenditure compared to the existing solutions.

\section{5) Entropy}

We use entropy to measure the path randomness. This metric captures the network traffic diversity, spread factor. More randomness in the routing paths followed by the packets more is privacy. Fig. 8 shows that the SPR technique has zero entropy and has the least entropy for the case of multiple asset scenarios. The two existing protocols, PRPRW and FRW, as well as our PRBRW have better entropy when compared to the SPR technique. But the entropy of PRBRW is the lowest when compared with PRPRW and FRW techniques. However, PRLPRW has the highest entropy indicating the highest privacy when compared to other protocols.

\section{6) Network Lifetime}

Another important metric we consider in this work is network lifetime. There's no free lunch. Privacy cannot be achieved without sacrificing energy expenditure. In our scenario, the network lifetime indicates the total number of packets sent by all the nodes in the network. This includes 


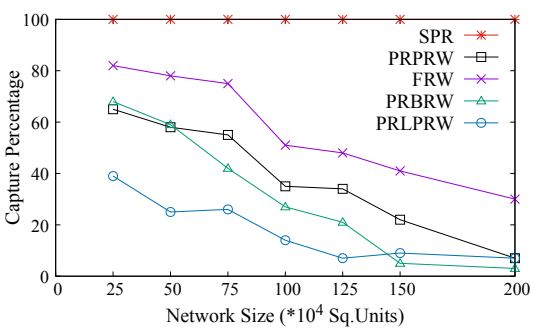

(a) Two Source Nodes in Opposite Quadrants

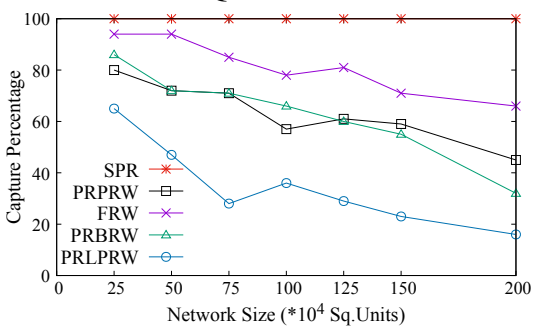

(d) Two Source Nodes in Same Quadrant

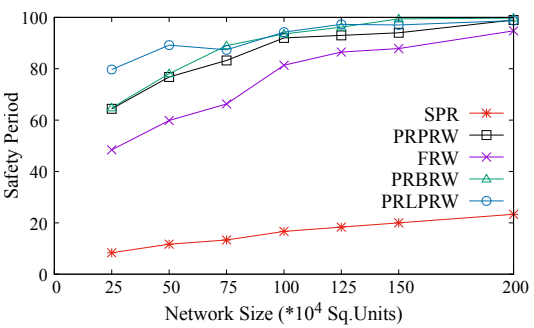

(b) Two Source Nodes in Opposite Quadrants

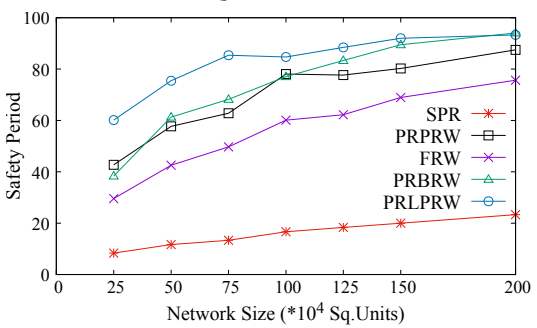

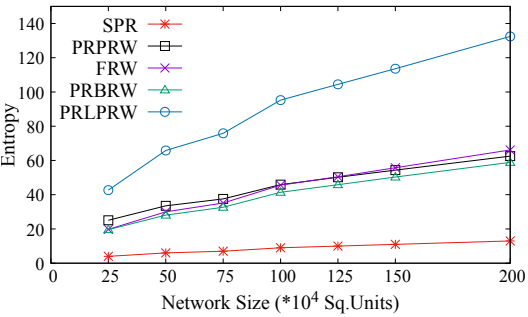

(c) Two source nodes in Opposite Quadrants

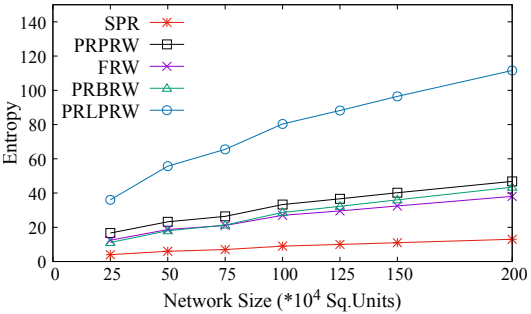

(e) Two Source Nodes in Same Quadrant (f) Two Source Nodes in Same Quadrant

Fig. 10: Performance Comparision of Two Source Nodes in Two Different Quadrants Case (Fig. 3b) with that of Two Source Nodes in Same Quadrant Case (Fig. 3e)

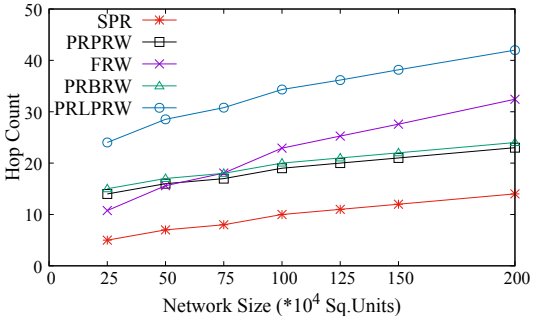

(a) Two Source Nodes in Opposite Quadrants

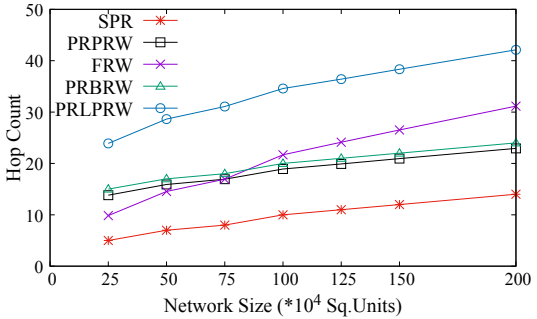

(d) Two Source Nodes in Same Quadrant

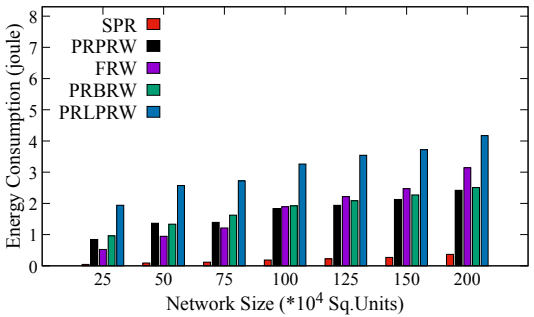

(b) Two Source Nodes in Opposite Quadrants

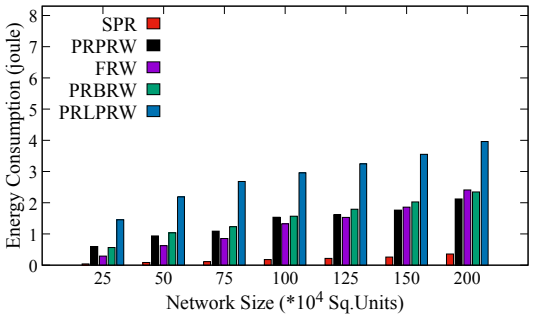

(e) Two Source Nodes in Same Quadrant

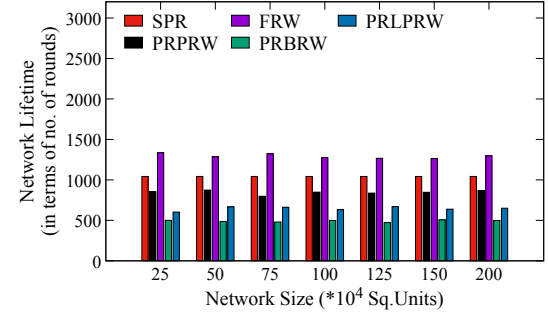

(c) Two Source Nodes in Opposite Quadrants

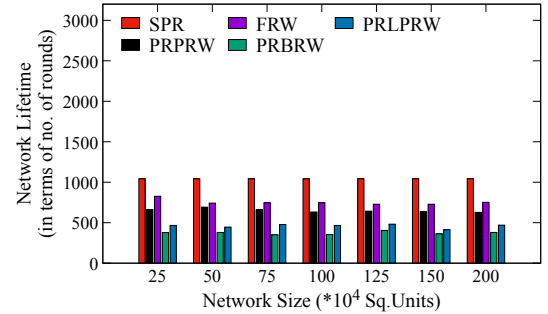

(f) Two Source Nodes in Same Quadrant

Fig. 11: Performance Comparision of Two Source Nodes in Two Different Quadrants Case (Fig. 3b) with that of Two Source Nodes in Same Quadrant Case (Fig. 3e)

right from the first packet sent to the BS up to a point where a node in the network dies, due to exhaustion of energy. This metric is as shown in Fig. 9. The network lifetime is the least in both the proposed techniques compared to PRPRW and FRW solutions.

\section{7) Two Source Nodes in the Same Quadrant}

We have also attempted to study the performance of the two source nodes lying in the same quadrant, almost close to each other (exact positions of these two source nodes are given in Tab. II). The capture ratio of two source nodes situated in opposite quadrants of the network has shown better performance. However, this is not true if they are close together, located in the same quadrant. The capture ratio for the same quadrant case is higher compared to that of the opposite quadrant case, this is shown in Fig. 10d and Fig. 10a respectively. The capture ratio is $100 \%$ for the SPR technique as expected and for other techniques, the capture ratio is lesser. The capture ratio is also influenced by the network size, and it decreases as the network size increases thus giving better privacy. Among the existing techniques, FRW performs poorly compared to PRPRW. In the proposed schemes, PRBRW performs well for larger network sizes whereas PRLPRW outperforms when compared to all other protocols for all the settings.

Fig. 10e and Fig. 10b shows the safety period metric for the same quadrant case and opposite quadrant case respectively. The safety period for the same quadrant case performs poorly 
TABLE III: Summary of Performance Comparison of SLP Techniques with no SLP, x-fold Improvement

\begin{tabular}{|l|l|l|l|l|l|l|}
\hline $\begin{array}{l}\text { Metrics vs. } \\
\text { Protocol }\end{array}$ & $\begin{array}{l}\text { Capture } \\
\text { Ratio }\end{array}$ & $\begin{array}{l}\text { Safety } \\
\text { Period }\end{array}$ & Entropy & $\begin{array}{l}\text { Hop } \\
\text { Count }\end{array}$ & $\begin{array}{l}\text { Energy } \\
\text { Consumption }\end{array}$ & $\begin{array}{l}\text { Network } \\
\text { Lifetime }\end{array}$ \\
\hline PRPRW & -54.92 & 433.01 & 316.62 & 104.24 & 971.54 & -24.44 \\
FRW & -34.96 & 341.44 & 288.80 & 123.33 & 820.72 & 11.10 \\
PRBRW & -60.39 & 455.25 & 265.00 & 116.21 & 1043.65 & -55.76 \\
PRLPRW & -73.17 & 483.98 & 703.57 & 266.54 & 2015.54 & -45.00 \\
\hline
\end{tabular}

when compared to the safety period of the opposite quadrant case. This decrease in performance in the same quadrant case is the direction of the packet flow towards the BS is almost confined to a single quadrant. It is easy for the attacker to narrow down its search space as traffic is coming from a single direction.

Fig. 10f and Fig. 10c shows the plots for the metric entropy. Larger is the entropy value more diverse or random are the routing paths. The entropy of SPR is the least, while PRPRW, FRW, and BRW have almost the same entropies. However, the entropy of the PRLPRW is the highest indicating the highest randomness in the packets' random paths. The entropy for the same quadrant scenario is lesser when compared to the entropy of the opposite quadrant scenario in the case of the PLRPRW technique. This is because in the opposite quadrant case the traffic from two sources takes different paths to reach the BS whereas in the same quadrant case, the traffic is flowing in almost the same quadrant leading to less routing path diversity. This observation suggests careful planning or designing of the routing protocols has to be done so that when multiple assets are close together, say in a crowd for food or shelter, the privacy level is not hampered.

The average number of hops for the same quadrant case and opposite quadrant case is depicted in Fig. 11d and Fig. 11a respectively. The hop count values in both scenarios remain the same. This is because the distance of the two source nodes from the BS in both the configuration is almost the same with a negligible difference. Hence the number of hops taken by packets also remains the same.

The energy expenditure that is incurred for delivering messages is given in Fig. 11e and Fig. 11b. The energy consumption is the highest in the PRLPRW technique when compared to all other protocols. This is due to great diversity and randomness in the paths traversed by the packets. The SPR has the least energy consumption. The energy consumption of FRW is lesser for small-sized networks and it increases as the network size increases. This is true for both scenarios. However, the energy consumption of the FRW technique is higher in the opposite quadrant case when compared to the same quadrant case. The energy consumption of the PRPRW technique is less than PRBRW for small size networks but both have almost the same energy expenditure for larger network sizes (in the case of opposite quadrant case). In the case of the same quadrant configuration, the energy consumption of PRPRW is always lesser compared to PRBRW for all the network sizes.

The network lifetime is smaller in the case of two source nodes located in the same quadrant when compared to the two source nodes located in two opposite quadrants. This is see in Fig. 11f and Fig. 11c respectively. The reduction in network lifetime for the same quadrant case is attributed to the fact that information packets from the two source nodes almost follow the same set of paths i.e., the paths in the quadrant where the sources are present. This implies the same set of nodes are expending energy to relay the packets from both the sources and thus drain their battery energy at a faster rate.

\section{8) Summary of the Results}

Summarizing the results, we present the improvements and limitations of the SLP preservation techniques w.r.t to no SLP scheme-Shortest Path Routing (SRP) scheme. These are as shown in Tab. III ${ }^{1}$.

The capture ratio shows negative values because it indicates the adversary's success ratio. Lower is the capture ratio better is the safety of the asset. The proposed techniques PRBRW and PRLPRW have 60 and 73 folds improvements over no SLP scheme whereas existing PRPRW and FRW have only 54 and 34 folds improvements respectively. Similarly, in terms of safety period and entropy, our proposed techniques perform better compared to no SLP (SPR) technique. But the entropy of PRBRW is lower than PRPRW and FRW.

The improvements in capture ratio, safety period and entropy comes at the expense of a large delay (expressed in terms of the number of hops) and the network lifetime metrics. FRW techniques have the best network lifetime while PRPRW, PRBRW, and PRLPRW have a smaller network lifetime. As claimed in Sec. I-C we see an improvement of 10 folds in the PRLPRW technique compared to the PRBRW solution. Although the network lifetime of the FRW technique is the best amongst the other SLP techniques, it performs poorly in terms of capture ratio, safety period, and entropy metrics. The limitation of our techniques over PRPRW and FRW is the reduced network lifetime.

\section{Discussion and Future Direction}

Although it is conventional to believe that presence of multiple source nodes in the network in itself provides better SLP, the simulation results demonstrated the contrary aspect. This fact was also mathematically shown in [26]. Therefore, we strongly feel that SLP techniques are required for WSN even if there are multiple sources in the network.

In subsection VII-B7, we have also given the emphasis on the performance of the SLP for two scenarios: i) Two source nodes are in different parts of the networks and ii) Two source nodes are co-located in a nearby place. This analysis shows that position of the source nodes w.r.t the BS also has an impact on the performance of the SLP techniques. In the future, the work on SLP can consider this point and analyze the performance.

Another interesting research direction is the study of SLP techniques for multiple source scenarios with mobile sources in the network. To date, the existing solutions have considered that the source node(s) are static in nature. The upcoming works could explore the case of multiple but mobile source scenarios. The work in [29] could be used to track the mobile source. However, the work aims at only tracking without privacy concerns.

\footnotetext{
${ }^{1} \mathrm{x}$ indicates the value of performance metrics
} 
A noteworthy observation we made in this work is the choice of privacy metric. For instance, in the same quadrant scenario with two sources, the FRW performs worst in terms of safety period and capture ratio metrics while its entropy measure is comparable to that of other techniques. Whereas in the case of multiple source scenarios with each source in different quadrants of the network, PRBRW performs quite well in terms of safety period and capture ratio. However, it performs poorly in terms of entropy metric. This observation directs us to think on the selection of the right choice of privacy metric for SLP preservation techniques.

As discussed in VII-B sections, improvement in privacy means the reduction in network lifetime. We need SLP solutions the could achieve improved privacy without hampering the network life-a joint privacy-network lifetime optimization problem.

To date, all the work in source location privacy have been developed and proven to be effective for the scenarios where sensors are active always. Nevertheless, it must be noted that to minimize the energy expenditure and enable prolonged network lifetime of the network, sleep/wake-up scheduling algorithms [30] are employed. The challenge is "How to track the targets in a privacy preserved manner if sensors are employing sleep/wake-up scheduling policies?". Research in this direction is more challenging.

\section{CONCLUSION}

In this work, we developed two random walk-based source location privacy-preserving techniques and studied their effectiveness, along with the existing techniques, in multi-asset settings. The proposed techniques PRBRW and PRLPRW have 60- and 73-fold improvement in terms of capture ratio when compared with protection-less scheme SPR, respectively. The study reveals that the presence of multiple sources in the network in itself do not provide location privacy and calls for developing privacy preserved routing techniques. We also observed that the position of the multiple sources, i.e., whether they are together or far apart, also has an impact on the performance of the solutions. An interesting observation we made in this work is that not all privacy-related metrics perform alike in terms of measuring the performance of the SLP techniques. What capture ratio and safety period metrics show as improvement may not be captured by entropy metric, which may show a degradation in the performance. Therefore, the choice of the right privacy metric has to be made carefully and it calls for a development of a generic framework to evaluate the performance of SLP protocols. Further, these protocols must be extended to scenarios where there are multiple but mobile sources. This type of real-life setting has received very little attention so for in monitoring assets using WSNs.

\section{APPENDIX A}

\section{AVERAGE HOP ESTIMATION}

The average number of hops in PRPRW is estimated as follows: The pure random walk in the first phase relays a packet to a distant node from the source for $h_{1}$ hops. The random walk may end in anyone of the circle as shown in Fig. 12. Let the distance between the source node (point c) and the point where the random walk terminated-phantom node (point a) - be $d_{3}$ units. Now we derive the formula to estimate the average number of hops between the source node and the base station. That is we must find the distance between the phantom node (point B) and the BS (point A), $d_{2}$.

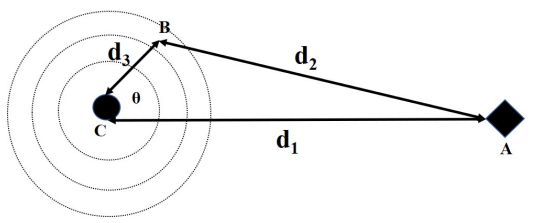

Fig. 12: Average Hop Estimation in PRPRW

Let the angle between $B C$ and $C A$ be denoted by $\theta$. The distance $d_{2}$ between point $\mathrm{B}$ and the base station (point $A$ ) is computed using the "law of cosines" and is given by

$$
\overline{d_{2}}\left(\theta, d_{3}\right)=\sqrt{d_{1}^{2}+d_{3}^{2}-2 d_{1} d_{3} \cos (\theta)}
$$

where $d_{2}$ is a function of both $\theta$ and $d_{3}$.

All the distances $d_{1}, d_{2}$ and $d_{3}$ and the sensor nodes radio range as well as the base station radio range, $r_{s}$, are in meters. Note: In terms of number of hops $d_{3}$ is expressed as $d_{3}=i * r_{s}$, $\forall \mathrm{i} \in 1,2,3, \cdots, h_{1}$.

Due to symmetry of the random walk in all the directions, $\theta$ is uniformly distributed between 0 and $2 \pi$. Therefore, the average distance while taking all directions into consideration is given by

$$
\overline{d_{2}}\left(d_{3}\right)=\frac{1}{2 \pi} \int_{0}^{2 \pi} \sqrt{d_{1}^{2}+d_{3}^{2}-2 d_{1} d_{3} \cos (\theta)} d \theta
$$

Simplifying this equation we have

$$
\overline{d_{2}}\left(d_{3}\right)=\sqrt{d_{1}^{2}+d_{3}^{2}} \quad(\text { in meters })
$$

or

$$
\overline{d_{2}}\left(d_{3}\right)=\frac{\sqrt{d_{1}^{2}+\left(i * r_{s}\right)^{2}}}{r_{s}} \quad(\text { in hops })
$$

The average distance between point $\mathrm{B}$ and the $\mathrm{A}$, given that $d_{3}=i * r_{s}$ is

$$
\left.\overline{d_{2}}=\frac{1}{h_{1}} \sum_{i=1}^{h_{1}} \sqrt{d_{1}^{2}+d_{3}^{2}} \quad \text { (in meters }\right)
$$

or

$$
\overline{d_{2}}=\frac{1}{h_{1}} \sum_{i=1}^{h_{1}} \frac{\sqrt{d_{1}^{2}+\left(i * r_{s}\right)^{2}}}{r_{s}} \quad \text { (in hops) }
$$

Therefore, the average number of hops $\bar{H}_{1}$ between the source node and the base station for PRPRW technique is given by

$$
\overline{H_{1}}=h_{1}+\frac{1}{h_{1}} \sum_{i=1}^{h_{1}} \frac{\sqrt{d_{1}^{2}+\left(i * r_{s}\right)^{2}}}{r_{s}}
$$

The average number of hops $\overline{H_{2}}$ in the froward random walk routing (FRW) technique [14] can be estimated using the following equation.

$$
\overline{H_{2}}=n^{\prime} / p_{r}=\left(d_{1} / r_{s}\right) / p_{r}
$$

where $n^{\prime}$ is the minimum number of hops between the source node and the base station and $p_{r}$ is the ratio of the number of neighbors at equal depth and number of neighbors at lower depth of node $n_{i}$ to the total number of neighbors of node $n_{i}$.

We now give the expression for the average number of hops between the source node and the base station of the proposed techniques. PRBRW has two components one is $h_{1}$ hops due to backward random walk and the second one is shortest path component. Since the backward random walk is half of the pure random walk with bias in opposite direction to the BS, 
the parameter $\theta$ now varies between $\frac{\pi}{2}$ and $\frac{3 \pi}{2}$. The distance between the phantom node and the BS $d_{2}$ is a function of $\theta$ alone. However, it also depends on number of hops $h_{1}$ in backward random walk that leads to the contribution of the distance $d_{3}\left(=r_{s} \times h_{1}\right)$ between source node and the phantom node. Therefore, $d_{2}$ is given by, using "Law of Cosines":

$$
\overline{d_{2}}(\theta)=\sqrt{d_{1}^{2}+d_{3}^{2}-2 d_{1} d_{3} \cos (\theta)} d \theta
$$

Due to bias in the random walk, $\theta$ is uniformly distributed between $\frac{\pi}{2}$ and $\frac{3 \pi}{2}$. Therefore, the average distance is given by

$$
\overline{d_{2}}=\frac{1}{\pi} \int_{\frac{\pi}{2}}^{\frac{3 \pi}{2}} \sqrt{d_{1}^{2}+d_{3}^{2}-2 d_{1} d_{3} \cos (\theta)} d \theta
$$

Simplifying this equation we have

$$
\overline{H_{3}}=\sqrt{d_{1}^{2}+d_{3}^{2}+\frac{4 d_{1} d_{3}}{\pi}} \quad \text { (in meters) }
$$

or

$$
\overline{H_{3}}=\frac{\sqrt{d_{1}^{2}+d_{3}^{2}+\frac{4 d_{1} d_{3}}{\pi}}}{r_{s}} \quad \text { (in hops) }
$$

Therefore, average number of hops $\overline{H_{3}}$ in case of PRBRW considering both the components of the routing phase is given by

$$
\overline{H_{3}}=h_{1}+\sqrt{d_{1}^{2}+d_{3}^{2}+\frac{4 d_{1} d_{3}}{\pi}} \quad \text { (in meters) }
$$

or

$$
\overline{H_{3}}=h_{1}+\frac{\sqrt{d_{1}^{2}+d_{3}^{2}+\frac{4 d_{1} d_{3}}{\pi}}}{r_{s}} \quad \text { (in hops) }
$$

where the first term in Eq. 10 represents the number of hops in BRW phase and the second term represents the number of hops in shortest path routing phase.

The average number of hops $\overline{H_{4}}$ in PRLPRW due to pure random walk is $h_{1}$ hops. The contribution due to L-path phase is $\left(\frac{L}{2 r_{s}}+\frac{L}{2 r_{s}}\right)$ hops attributing to vertical and horizontal walks respectively. Finally, the last contribution towards hops due to shortest path phase is $\frac{L}{r_{s} \sqrt{2}}$. Here, $L$ is the length of the deployment area. Summing up all these together we have:

$$
\begin{array}{r}
\bar{H}_{4}=h_{1}+\left(\frac{L}{2 r_{s}}+\frac{L}{2 r_{s}}\right)+\frac{L}{r_{s} \sqrt{2}} \\
=h_{1}+\frac{2 L}{2 r_{s}}+\frac{L}{r_{s} \sqrt{2}} \\
=h_{1}+\frac{L}{r_{s} \sqrt{2}}(\sqrt{2}+1)
\end{array}
$$

\section{REFERENCES}

[1] T. B. D. of ELECTRICAL and C. ENGINEERING, "Laying groundwork for the Internet of Battlefield Things,"

https://ece.vt.edu/news/article/the-internet-of-battlefield-things , Jan 2017, [Online; accessed 17-Dec-2020].

[2] K. Polit, "Army Takes on Wicked Problems With the Internet of Battlefield Things,"

https://www.meritalk.com/articles/army-takes-on-wicked-problemswith-the-internet-of-battlefield-things/,

Jan 2018, [Online; accessed 17-Dec-2020].

[3] J. J. Kang, W. Yang, G. Dermody, M. Ghasemian, S. Adibi, and P. Haskell-Dowland, "No soldiers left behind: An iot-based low-power military mobile health system design," IEEE access, vol. 8, pp. 201498 $201515,2020$.
[4] P. TUCKER, "US Army Seeks Internet-of-Battlefield-Things, Distributed Bot Swarms,"

https://www.defenseone.com/technology/2017/07/facial-recognitioncoming-police-body-cameras/139472/,

Aug. 2017, [Online; accessed 17-Dec-2020].

[5] C. Osborne, "US Army tests IoT for the battlefield in smart cities," https://www.zdnet.com/article/us-army-tests-iot-for-the-battlefield-insmart-cities/,

Aug. 2019, [Online; accessed 17-Dec-2020].

[6] K. E. Friedl, "Military applications of soldier physiological monitoring," Journal of science and medicine in sport, vol. 21, no. 11, pp. 1147-1153, 2018.

[7] A. Sternstein, "A more connected military means new battlefield glitches, too,"

https://www.csmonitor.com/World/Passcode/2017/0331/A-moreconnected-military-means-new-battlefield-glitches-too , March 2017, [Online; accessed 17-Dec-2020].

[8] R. Danzig, Surviving on a Diet of Poisoned Fruit: Reducing the National Security Risks of America's Cyber Dependencies. Center for a New American Security, 2014.

[9] N. Li, N. Zhang, S. K. Das, and B. Thuraisingham, "Privacy preservation in wireless sensor networks: A state-of-the-art survey," Ad Hoc Networks, vol. 7, no. 8, pp. 1501-1514, 2009.

[10] M. Conti, J. Willemsen, and B. Crispo, "Providing source location privacy in wireless sensor networks: A survey," IEEE Communications Surveys \& Tutorials, vol. 15, no. 3, pp. 1238-1280, 2013.

[11] C. Ozturk, Y. Zhang, and W. Trappe, "Source-location privacy in energyconstrained sensor network routing," in Proceedings of the 2nd ACM workshop on Security of ad hoc and sensor networks. ACM, 2004, pp. 88-93.

[12] W. P. Wang, L. Chen, and J. . Wang, "A source-location privacy protocol in wsn based on locational angle," in 2008 IEEE International Conference on Communications, 2008, pp. 1630-1634.

[13] Y. Tscha, "Routing for enhancing source-location privacy in wireless sensor networks of multiple assets," Journal of Communications and Networks, vol. 11, no. 6, pp. 589-598, 2009.

[14] H. Chen and W. Lou, "On protecting end-to-end location privacy against local eavesdropper in wireless sensor networks," Pervasive and Mobile Computing, vol. 16, pp. 36-50, 2015.

[15] M. Raja and R. Datta, "An enhanced source location privacy protection technique for wireless sensor networks using randomized routes," IETE Journal of Research, vol. 64, no. 6, pp. 764-776, 2018.

[16] R. Manjula and R. Datta, "A novel source location privacy preservation technique to achieve enhanced privacy and network lifetime in wsns," Pervasive and Mobile Computing, vol. 44, pp. 58-73, 2018.

[17] C. Ozturk, Y. Zhang, W. Trappe, and M. Ott, "Source-location privacy for networks of energy-constrained sensors," in Second IEEE Workshop on Software Technologies for Future Embedded and Ubiquitous Systems, 2004. Proceedings., 2004.

[18] K. Mehta, D. Liu, and M. Wright, "Protecting location privacy in sensor networks against a global eavesdropper," IEEE Transactions on Mobile Computing, vol. 11, no. 2, pp. 320-336, 2011.

[19] Y. Yang, M. Shao, S. Zhu, and G. Cao, "Towards statistically strong source anonymity for sensor networks," ACM Transactions on Sensor Networks (TOSN), vol. 9, no. 3, pp. 1-23, 2013.

[20] G. Han, X. Miao, H. Wang, M. Guizani, and W. Zhang, "Cpslp: A cloud-based scheme for protecting source location privacy in wireless sensor networks using multi-sinks," IEEE Transactions on Vehicular Technology, vol. 68, no. 3, pp. 2739-2750, 2019.

[21] N. Wang, J. Fu, J. Li, and B. K. Bhargava, "Source-location privacy protection based on anonymity cloud in wireless sensor networks," IEEE Transactions on Information Forensics and Security, vol. 15, pp. 100$114,2019$.

[22] J. Y. Koh, G. W. Peters, I. Nevat, and D. Leong, "Probabilistic routing in wireless networks with privacy guarantees," Computer Communications, vol. 151 , pp. $228-237,2020$

[23] Rimjhim, P. K. Roy, and J. P. Singh, "Finding location of fake and phantom source for source location privacy in wireless sensor network," International Journal of Communication Networks and Distributed Systems, vol. 26, no. 1, pp. 114-134, 2021.

[24] J. F. Laikin, M. Bradbury, C. Gu, and M. Leeke, "Towards fake sources for source location privacy in wireless sensor networks with multiple sources," in 2016 IEEE International Conference on Communication Systems (ICCS). IEEE, 2016, pp. 1-6.

[25] M. Bradbury, M. Leeke, and A. Jhumka, "A dynamic fake source algorithm for source location privacy in wireless sensor networks," in 2015 IEEE Trustcom/BigDataSE/ISPA, vol. 1. IEEE, 2015, pp. 531538.

[26] H. Wang, B. Sheng, and Q. Li, "Privacy-aware routing in sensor networks," Computer Networks, vol. 53, no. 9, pp. 1512-1529, 2009.

[27] Z. Kashino, G. Nejat, and B. Benhabib, "A hybrid strategy for target search using static and mobile sensors," IEEE Transactions on Cybernetics, vol. 50, no. 2, pp. 856-868, 2020. 
[28] W. B. Heinzelman, A. P. Chandrakasan, and H. Balakrishnan, "An application-specific protocol architecture for wireless microsensor networks," IEEE Transactions on wireless communications, vol. 1, no. 4, pp. 660-670, 2002.

[29] H. Mahboubi, W. Masoudimansour, A. G. Aghdam, and K. SayrafianPour, "An energy-efficient target-tracking strategy for mobile sensor networks," IEEE Transactions on Cybernetics, vol. 47, no. 2, pp. 511523,2017

[30] D. Ye and M. Zhang, "A self-adaptive sleep/wake-up scheduling approach for wireless sensor networks," IEEE Transactions on Cybernetics, vol. 48, no. 3, pp. 979-992, 2018. 